\title{
THE IONISATION EQUILIBRIUM FOR HEAVY ELEMENTS
}

\author{
D. R. FLOWER \\ (Dept. of Physics, University College London, England)
}

\begin{abstract}
Calculations are being made of the distribution of the ions of heavy elements in planetary nebulae. Initial work has been concentrated on the central or $\mathrm{He}^{2+}$ zone of planetaries. The optical depths of ions of $\mathrm{C}, \mathrm{N}, \mathrm{O}$, and $\mathrm{Ne}$ have been computed using ground state ionisation cross-sections and using approximations which should be substantially better than hydrogenic. A comparison has been made between the combined optical depth of the heavy elements and the optical depth of $\mathrm{He}^{+}$in the far ultraviolet. The optical depths of the heavy elements in this spectral region may become significant, but a reasonable first approximation to the radiation field may be obtained by neglecting the absorption of all ions except $\mathrm{He}^{+}$. The distribution of the ions of the heavy elements has been calculated on this assumption.
\end{abstract}

Calculations have been made of the distribution of ions of heavy elements in the central zone of planetary nebulae. The basic problem is to solve the ionisationequilibrium equation at each point in the central zone. If we consider an element $X$, the equilibrium between the $q$ th and $(q+1)$ th stages of ionisation may be written

$$
4 \pi N\left(X^{q+}\right) \int J_{v} a_{v} \mathrm{~d} v=N_{\mathrm{e}} N\left(X^{(q+1)+}\right) \alpha .
$$

$4 \pi J_{v}$ is the mean flux of photons, $N$ represents a number density, $N_{\mathrm{e}}$ is the electron density, $a_{v}$ the ground state photo-ionisation cross-section, and $\alpha$ is the total radiative recombination coefficient. If the optical depth associated with the ion $X^{q+}$ is assumed to be small, then the total recombination coefficient is a sum of coefficients for radiative recombination to all excited states and to the ground state:

$$
\alpha=\sum_{n=n^{\prime}}^{\infty} \alpha_{n}
$$

where $n^{\prime}$ is the principal quantum number of the ground state of the ion $X^{q+}$. The recombination coefficients are slowly varying functions of the electron temperature, $T_{\mathrm{e}}$. If equations of the form of (1) can be solved, then the ratio, $N\left(X^{(q+1)+}\right) / N\left(X^{q+}\right)$, of the concentrations of successive stages of ionisation of the element $X$ can be obtained at each point in the central zone of the nebula. Given the abundance of $X$ relative to hydrogen, it is then possible to calculate the abundance of each ion of $X$ relative to hydrogen at each point.

Initial calculations have been restricted to the central ionisation zone of planetaries

Osterbrock and O'Dell (eds.), Planetary Nebulae, 205-208. (I.A.U. 
where most of the helium is fully ionised, i.e., most of the helium is $\mathrm{He}^{2+}$. This zone will be a significant fraction of the total nebular volume only in high-excitation nebulae. All the ions present in this region of the nebula will have ionisation potentials above the $\mathrm{He}^{+}$threshold value at $228 \AA$. The radiation field shortwards of $228 \AA$ is calculated at each point of the central zone on the assumption that $\mathrm{He}^{+}$dominates the absorption of this radiation in the central region. This would appear to be a reasonable first approximation because the photo-ionisation cross-section of hydrogen is very small at such short wavelengths and the abundance of helium is much greater than the abundances of heavier elements. Essentially, we assume initially that only $\mathrm{He}^{+}$contributes significantly to the optical depth in the central zone. The photon flux at some point in the central zone distant $R$ from the nuclear star is then given by

$$
4 \pi J_{v}=\frac{L_{v}}{4 \pi R^{2}} e^{-\tau_{v}\left(\mathrm{He}^{+}\right)}
$$

on a spherically symmetric model. $\tau_{v}\left(\mathrm{He}^{+}\right)$is the optical depth of $\mathrm{He}^{+}$at frequency $v$, and $L_{v}$ the photon luminosity of the star at that frequency.

The ground-state photo-ionisation cross-sections, $a_{v}$, required for a solution of Equation (1) are taken from a paper by Seaton (1958) for ions with electron configurations $1 \mathrm{~s}^{2} 2 \mathrm{~s}^{2} 2 \mathrm{p}^{q}$, where $q$ is any integer from 1 to 6 inclusive. Seaton fits his calculations to a function of the form

$$
a_{v}=B C \times 10^{-18}\left\{\alpha\left(\begin{array}{c}
v \\
v_{0}
\end{array}\right)^{-s}+(1-\alpha)\left(\begin{array}{c}
v \\
v_{0}
\end{array}\right)^{-s-1}\right\}
$$

where $B, C, s$, and $\alpha$ are tabulated quantities, $v_{0}$ is the ionisation threshold frequency, and $a_{v}$ is in units of $\mathrm{cm}^{2}$. Similar calculations have been completed for the configurations $1 s^{2} 2 s$ and $1 s^{2} 2 s^{2}$, and the results are presented in Tables 1 and 2 . The calculations were based on the method of quantum defects described by Burgess and Seaton (1960) using revised tabulations of necessary functions given by Peach (1967). The calculations for the $1 s^{2} 2 s$ configuration presented no difficulty and the results should be substantially better than hydrogenic approximations. Calculations for the

Table 1

Photo-ionisation cross-section parameters for the lithium sequence, $1 \mathrm{~s}^{2} 2 \mathrm{~s}$, for which $C=1$. The cross-section is then given by Equation (4)

Ion

$\mathrm{Li}$

$\mathrm{Be}^{+}$

$\mathrm{B}^{2+}$

$\mathrm{C}^{3+}$

$\mathrm{N}^{4+}$

$\mathrm{O}^{5+}$

$$
B
$$

1.3

1.4

0.98

0.68

0.48

0.36

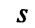

$1 \cdot 4$

2.0

$1 \cdot 8$

2.0

2.0

$2 \cdot 1$ $\alpha$

$3 \cdot 5$

$2 \cdot 2$

$1 \cdot 0$

1.0

1.0

1.0 


\section{Table 2}

Photo-ionisation cross-section parameters for the beryllium sequence, $1 \mathrm{~s}^{2} 2 \mathrm{~s}^{2}$, for which $\mathrm{C}=2$. The cross-section is then given by Equation (4). $\mathrm{Be}^{0}$ is omitted because the results obtained were not accurate for that particular case

\begin{tabular}{llll} 
Ion & \multicolumn{1}{c}{$B$} & $s$ & $\alpha$ \\
& & & \\
$\mathrm{B}^{+}$ & 1.5 & $3 \cdot 0$ & $2 \cdot 6$ \\
$\mathrm{C}^{2+}$ & $0 \cdot 80$ & $3 \cdot 0$ & $2 \cdot 6$ \\
$\mathrm{~N}^{3+}$ & 0.54 & $3 \cdot 0$ & $2 \cdot 6$ \\
$\mathrm{O}^{4+}$ & 0.39 & $3 \cdot 0$ & $2 \cdot 6$ \\
$\mathrm{~F}^{5+}$ & 0.29 & 3.0 & 2.6
\end{tabular}

$1 s^{2} 2 s^{2}$ sequence are complicated by the presence of equivalent electrons in the outer shell. The results presented are accurate only at threshold and the frequency variation is taken to be hydrogenic. The results for the neutral in the $1 s^{2} 2 s^{2}$ sequence, namely $\mathrm{Be}^{0}$, were poor and have been omitted from Table 2 . Nevertheless, figures presented for higher members of this sequence should be significantly better than hydrogenic approximations.

Having solved for the distribution of ions of the heavy elements in the central zone it is then possible to calculate the optical depths of these ions and compare their combined optical depth with that of $\mathrm{He}^{+}$. At wavelengths just shortwards of $228 \AA$ the optical depth of $\mathrm{He}^{+}$will indeed be much greater than the combined optical depth of other ions. However, as Khromov (1967) has pointed out, this may not be the case at much shorter wavelengths because the ground-state photo-ionisation cross-section of $\mathrm{He}^{+}$decreases inversely as the third power of the frequency. In the far ultraviolet the combined optical depth of ions of the heavy elements may become comparable with or even greater than that of $\mathrm{He}^{+}$.

Table 3 shows the results of calculations of optical depths at $90 \AA$. The inner radius of the nebular shell is taken as $5 \times 10^{15} \mathrm{~cm}$ and the central star is assumed to radiate

Table 3

Optical depths of $\mathrm{He}^{+}$and ions of heavier elements at a wavelength of $90 \AA$. The absolute hydrogen density is taken as $1.0 \times 10^{4} \mathrm{~cm}^{-3}$ and the relative element abundances are taken from Aller (1964)

\begin{tabular}{|c|c|c|c|c|c|c|c|}
\hline \multirow{2}{*}{$\begin{array}{c}\text { Radius } \\
\left(\times 10^{-16} \mathrm{~cm}\right)\end{array}$} & \multirow{2}{*}{$\begin{array}{c}\text { Optical } \\
\text { depth of } \\
\mathrm{He}^{+} \text {at } \\
90 \AA\end{array}$} & \multicolumn{5}{|c|}{ Optical depths of ions of the heavy elements at $90 \AA$} & \multirow{2}{*}{$\begin{array}{l}\text { Combined } \\
\text { optical depth } \\
\text { of ions } \\
\text { of the heavy } \\
\text { elements }\end{array}$} \\
\hline & & $\mathrm{C}^{3+}$ & $\mathrm{N}^{4+}$ & $\mathrm{O}^{4+}$ & $\mathrm{Ne}^{3+}$ & $\mathrm{Ne}^{4+}$ & \\
\hline $4 \cdot 5$ & $0 \cdot 120$ & 0.005 & 0.017 & $0 \cdot 134$ & 0.073 & 0.111 & 0.340 \\
\hline $5 \cdot 0$ & 0.229 & 0.008 & 0.018 & $0 \cdot 142$ & 0.093 & $0 \cdot 118$ & 0.379 \\
\hline $5 \cdot 5$ & 0.632 & 0.011 & 0.019 & 0.145 & 0.112 & 0.121 & 0.408 \\
\hline $5 \cdot 81$ & 1.201 & 0.013 & 0.019 & 0.146 & 0.118 & 0.122 & 0.418 \\
\hline
\end{tabular}


as a black body at $12 \times 10^{4}{ }^{\circ} \mathrm{K}$. The electron temperature of the central zone is assumed constant at $2 \times 10^{4}{ }^{\circ} \mathrm{K}$ (Flower, 1968). It appears that absorption of far-ultraviolet radiation by ions of the heavy elements is at least as important as absorption by $\mathrm{He}^{+}$. More refined models of the ionisation structure of planetary nebulae should take this absorption into account.

\title{
Acknowledgements
}

This work was completed whilst the author was in tenure of a Science Research Council studentship. The author would like to thank Professor M.J. Seaton for his interest in this work.

\section{References}

\author{
Aller, L.H. (1964) Astrophys. Norw., 9, 293. \\ Burgess, A., Seaton, M.J. (1960) Mon. Not. R. astr. Soc., 120, 121. \\ Flower, D.R. (1968) in the present volume, p. 77. \\ Khromov, G.S. (1967) Mon. Not. R. astr. Soc., 137, 175. \\ Peach, G. (1967) Mem. R. astr. Soc., 71, 13. \\ Seaton, M.J. (1958) Rev. Mod. Phys., 30, 979.
}

